\title{
Real-time Earthquake Location Using Kirchhoff Reconstruction
}

\author{
by Teresa Baker, Robert Granat, and Robert W. Clayton
}

\begin{abstract}
Real-time location of earthquakes can be achieved by using direct imaging of the recorded wave field based on a Kirchhoff reconstruction method similar to that used in the migration of seismic reflection data. The standard method of event location requires the wave arrival at each sensor to be picked and associated with an event. By using direct imaging, the event is identified once in the imaged wave field. The computation is independent of the level of seismic activity and can be carried out on a typical desktop computer. The procedure has been successfully demonstrated in two and three dimensions using data from the Southern California Seismic Network (Trinet). At higher resolutions, the reconstruction method can identify finite source effects. Further work considers extending the method by implementing full elastic theory and solving for moment tensors at all locations in the mesh.
\end{abstract}

\section{Background}

Routine earthquake location done with most seismic arrays is based on some form of Geiger's method (Udias, 1999), which locates the hypocenter by minimizing the error between the predicted and observed travel times to each station in the array. This means the individual channels in the array need to be "picked" and these picks need to be associated with a specific seismic phase of a potential event. The association step involves some iteration when multiple events are occurring and, in the case of a major earthquake, the aftershocks can often overwhelm this process, thus making it difficult to keep up with real time. Furthermore, there is a serious problem of picking arrivals when they fall in the coda of a larger event. A common practice is to stop picking for some period after an event to prevent false coda picks. This creates shadow zones of events after a large event (Vidale et al., 2003). The essential problem is that picking is done on a "per seismogram" basis and makes no use of the coherency of the phases between stations.

The accuracy of event location has been improved by using the double-differencing algorithm presented by Waldhauser and Ellsworth (2000) and by using cross-correlation to measure the relative travel-time delays at the stations (Richards-Dinger and Shearer, 2000). Global search algorithms have made it computationally practical to use probabilistic earthquake location and maintain a complete description of the uncertainties contributing to the solution (Lomax, 2000; Husen et al., 2003). These methods, however, are not well suited to real-time applications, because, in general, they do not deal with the association problem and hence are sensitive to interference by other earthquakes.

In this article we demonstrate an alternative location procedure for use with dense seismic networks which involves no picking of the seismograms. It is based on a Kirchhoff reconstruction of the ground motions on a mesh from the seismograms recorded by the network. Kirchhoff migration is commonly used to process data in reflection seismology for structural imaging. In this case, the reflected energy is distributed over a number of receivers, and migration moves the reflection events back to the actual source points, resulting in an image of the subsurface structure. The technique uses a surface integral of the scalar wave equation over the seismic observations that form the boundary values (Schneider, 1978). Further developments have broadened the uses of migration to include velocity estimation and attribute analysis in addition to structural imaging. In the earthquake application, migration is used to focus energy received at several stations back to the earthquake source.

Several techniques exist for locating earthquake sources that are related to Kirchhoff migration. These include using continuous waveform data to locate reflectors, reverse-time migration, and a source-scanning algorithm. Reverse-time migration, which is similar to Kirchhoff migration, has been investigated by McMechan and colleagues (1985) as a method for imaging earthquake sources. The sensor data are interpolated onto a grid and the wave equation is tracked in reverse time to extrapolate waves back to the source. Predetermined velocity structures were required and a finitedifference method was used; testing was performed on three events in Long Valley Caldera, 1983. This approach was later extended to incorporate all three waveform components (Chang and McMechan, 1991). Most recently, the sourcescanning algorithm (SSA) was developed by Kao and Shan (2004) and tested by locating tremors that occurred during the early 2003 swarm under Cascadia. The similarities of this method to the Real-time Kirchhoff Location (RKL) method presented in this article are discussed in the next section. 


\section{Approach}

The RKL method images ground motion continuously, allowing earthquake hypocenter locations to be identified as "points" in the images. The images are calculated on a mesh over the area of interest. The contributing waveform data can be observed by any station geometry, including stations inside or outside of the mesh boundaries. Motion recorded at the seismic station is projected onto the mesh at candidate source locations. Large values at a mesh point indicate coherent summation from many of the contributing stations and hence the location of an event.

The method offers several new capabilities. The earthquake locations are calculated in real time, without phase picking and associating required to identify the data for a particular event. A fixed computational load is maintained independent of the number of events occurring or the event magnitudes. Events will cause peaks at the correct locations in the reconstruction image, regardless of the size of other nearby events. It is possible to incorporate subsurface material properties of arbitrary complexity with no additional run-time computational overhead. Increased complexity would allow, for instance, additional Green's functions to represent scatterers, such as basin boundaries and the Moho. With increasing mesh resolution, finite-source effects will be observable in real time, which will help in identifying the rupture plane.

The RKL method is designed to be integrated with large seismic arrays for real-time monitoring. Specifically, it is to be a robust location estimator that will not fail during an event because of increased computational load placed on the system. The initial candidate array for the RKL method is the TriNet system in southern California, which maintains 155 broadband stations (Jones et al., 2003).

The Kirchhoff migration used by the RKL method is different from the SSA presented by Kao and Shan (2004) in several respects. In contrast to the SSA, the RKL method has been developed to run continuously in real time. The method does not require storage of sensor recordings before or after the current time step, whereas the SSA requires a time window of sensor data that is as long as the maximum station to mesh-point travel time. Additionally, because SSA is designed to scan efficiently over a fixed period assumed to contain an event, one station is chosen as the reference station and the brightness is only calculated at points that could be source locations for the largest arrival in the waveform at that reference station. The RKL method is implemented by using a $P$-wave velocity model, resulting in locations corresponding to the coherent summing of $P$-wave arrivals. SSA calculates wave travel times based on the speed of the wave with the largest amplitude, generally the $S$ wave. $S$ waves travel more slowly, delaying the calculation of the event location, which is an important consideration for earlywarning and rapid-response systems.

\section{Theory}

An acoustic theory is used to model $P$ waves that propagate through the earth from an earthquake source. The model works well for predicting $P$-wave amplitude and speed. The acceleration recorded at each sensor is a function of time, the location of the receiver, and the contributing sources, which include environmental noise and earthquakes. In the simplest construction, the receiver recording represents the one-way travel time from a source.

The wave equation is reversible in time and space and in reflection seismology this property is used by Kirchhoff migration to downward continue the field. In the RKL method, the field is "backward" continued from the receiver. For a homogeneous velocity model, the continued field falls on a spherical shape around the recording point. The amplitude of the signal at each time and each receiver is added to all the continued points. A source is resolved when the migration of all the receiver signals converge. Shear and converted waves travel at a different velocity and therefore do not add coherently in reconstructions based on the $P$-wave velocity. The output of this migration process is an image of the amplitude of the correlated energy at each point in a mesh covering the area of interest. Peak values occur where the migrated receiver signals add coherently.

To implement this theory, a sum of the appropriate signal contributions is performed at each mesh point at each time step:

$$
K_{i}(t)=\sum_{n=1}^{N} W_{n}\left(t+\left\|\mathbf{s}_{n}-\mathbf{m}_{i}\right\| / v\right)
$$

where $K_{i}(t)$ is the value of the reconstruction at time $t$ and mesh point $i$ with coordinate vector $\mathbf{m}_{i}=\left(x_{i}, y_{i}, z_{i}\right), W_{n}(t)$ is the magnitude of the ground-motion waveform recorded by sensor $n$ at vector coordinate $\mathbf{s}_{n}$ and time $t, v$ is the wave velocity, and $N$ is the number of seismic sensors. In equation (1) a uniform velocity $v$ is assumed. The algorithm can be carried out in either of two entirely equivalent forms, image based and data based. In the image-based form, for a given image point, all the appropriate data are summed. In the databased form, each data point is composited to all the possible image points. This is implemented by adding the current waveform value to the sets of points for each possible travel time in an array of frames. Pseudocode of the implementation is presented in Listing 1. This data-based implementation is used for the experiments presented here.

A table of travel times is used to increase the efficiency of the method by eliminating repetitive distance calculations. The table can either be developed by deterministic methods (ray-tracing, finite-difference) applied to a specific velocity model or by empirical travel-time curves. In the latter case, the tables are determined by using a set of travel times that best fit the locations of a suitable set of "standard events." 
Listing 1

Pseudocode for the Kirchhoff Reconstruction Algorithm

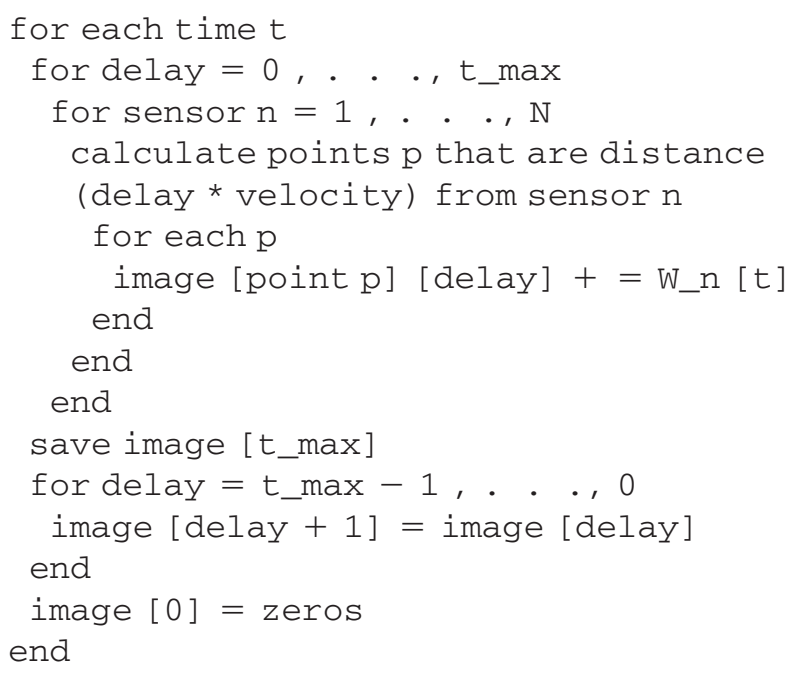

This table can also be used to incorporate data corresponding to the arrival of waves reflected off local structures to improve the resolution of the reconstruction. The mesh-point sensor pairs can be cross-correlated with a predetermined signal representing the seismogram for an earthquake occurring at that mesh point. The predetermined signal can include additional phases and reflected arrivals.

Because acoustic theory does not account for the double couple associated with a real earthquake rupture, all the waveforms used must be converted to positive amplitudes for the purposes of location. This is achieved by using an envelope function, which preserves the frequencies of the original signal. (Note: the SSA chooses to use the absolute value of waveforms with normalized amplitudes instead.)

The ground motion that occurs at a particular time at each point in the area of interest is not recorded concurrently at all the sensors. To implement the reconstruction in real time, all the data recorded by the sensors for the current time step is stored in a set of matrices corresponding to prior time steps. Hereafter, these matrices of the mesh-point values are referred to as frames. The time difference between each frame and the current recording determines which mesh points the recorded motion could have traveled from in that frame. The data are thus added to a different set of mesh points in every frame. The information in the frames is shifted to the prior time slot after each recording and data for the new time step are added to the frames. The maximum delay frame is output at the end of each time step because it is complete, containing the motion recorded from every sensor. One can imagine during this process that the image frames are on a circular rack. At each time step the rack rotates, and the most complete image that has received all possible sensor waveform contributions is recorded, cleaned, and then moved to the front of the rack. When the recon- struction is run continuously, the maximum delay frames can be viewed as a movie of the real-time ground motion.

The implementation of the RKL method scales computationally as

$$
O\left(N r t_{\max }\right)
$$

where $N$ is the number of sensors, $t_{\max }$ is the maximum travel time, and $r$ is the mesh resolution. The maximum travel time is calculated as

$$
t_{\max }=\max _{i, n}\left\|\mathbf{s}_{n}-\mathbf{m}_{i}\right\| / v .
$$

The time per frame scales linearly with the number of sensors used in the reconstruction. The time per frame also scales linearly with the maximum travel-time delay between any mesh point and sensor pair because this delay determines the number of frames maintained for the reconstruction. At every time step, the current sensor recordings are added to each of the reconstruction frames. Increased mesh resolution also increases the computation time per frame. The computation can scale sublinearly with resolution because it scales with the number of points in the mesh that are an equal travel time from the sensor. This is a major advantage of the approach because it means that computation is not directly proportional to the total number of mesh points. The computation time per frame is independent of the number of events occurring, the magnitude of the events occurring, and the complexity of the velocity and reflector models.

Note that the complete reconstruction of each frame is only achieved after a delay. This delay time is proportional to the travel time of the waves of interest across the network plus the network latency.

\section{Tests}

The RKL method is designed to efficiently handle data from a large array of sensors and perform a solution over a regional mesh area. To test the robustness and the reliability of the method, three experiments were performed with the existing Trinet station geometry and actual events, and a fourth experiment using simulated waveforms was used to show the ability of the method to locate a rupture fault. For the first three experiments, $z$-component waveforms downloaded from the Southern California Earthquake Data Center (SCEDC) (2004) database were used. The chosen events were all greater than 4.0 magnitude, with the best available location quality. The waveforms were shifted to account for individual station delays and filtered to remove environmental noise and poor station recordings. An envelope function was applied to each signal, as described in the Theory section. Solutions were calculated on a 3D mesh covering the Los Angeles basin, with all locations relative to the north- 
west corner of the mesh. Figure 1 shows the mesh boundary and Trinet station locations overlying California. A uniform $P$-wave velocity of $6 \mathrm{~km} / \mathrm{sec}$ was assumed for these experiments.

To demonstrate that the RKL method can handle events occurring close in time, the reconstruction was run with a set of waveforms created by superimposing an event on top of the same event shifted $10 \mathrm{sec}$ forward in time. The waveforms recorded by 113 broadband stations for a $M 4.1$ event two miles east-northeast of San Fernando, California, on 14 January 2001 were used. The reconstruction found two peaks with similar shapes separated by $10 \mathrm{sec}$ (200 frames) (shown in Fig. 2). The peaks were both located less than 1 $\mathrm{km}$ from the true event location. The magnitude of the second peak is larger than the initial peak because it is augmented by the migration of data from the original event.

The RKL method is also robust to events occurring close together in space. To demonstrate this capability, waveforms from the same event east-northeast of the city of San Fernando were superimposed on a set of identical waveforms, shifted to create an apparent event location $46 \mathrm{~km}$ to the east and $10 \mathrm{~km}$ to the south. The time shift applied to each individual frame was calculated as the change in travel time between the sensor and the event location. The reconstruction image at the time of the peak value is shown in Figure
3. The two highest valued points in the mesh are located at the expected event locations. Surrounding the points are patterns of intersecting rings, which are the result of incomplete sampling of the wave field.

To test the reliability of the method, 40 real event locations were reconstructed. Figure 4 shows the expected event locations within the mesh along with the locations found by the reconstruction. The event locations found by the reconstruction are identified by choosing the reconstruction frame containing the peak value across all frames and extracting the location of that peak value. Spikes due to noise are minimal in the reconstruction because the noise does not add coherently over all the stations. Figure 5 shows the accuracy of the solutions, with $80 \%$ of the events located within $10 \mathrm{~km}$ of the true event locations. As an example, with a Linux workstation with a 2.2-GHz Opteron processor the time per frame for this calculation is $0.35 \mathrm{sec}$. The calculation is on a $225 \times 200$ grid and includes 113 sensors.

The solution resolution of this set of tests is not high enough to identify an entire rupture fault by using real waveforms. Nevertheless, the capability of the method can be demonstrated by using synthetic data. Figure 6 shows the peak reconstruction frame for a simulated event in the Trinet station geometry. The reconstruction images were calculated over the same mesh used in the previous experiments. Wave-

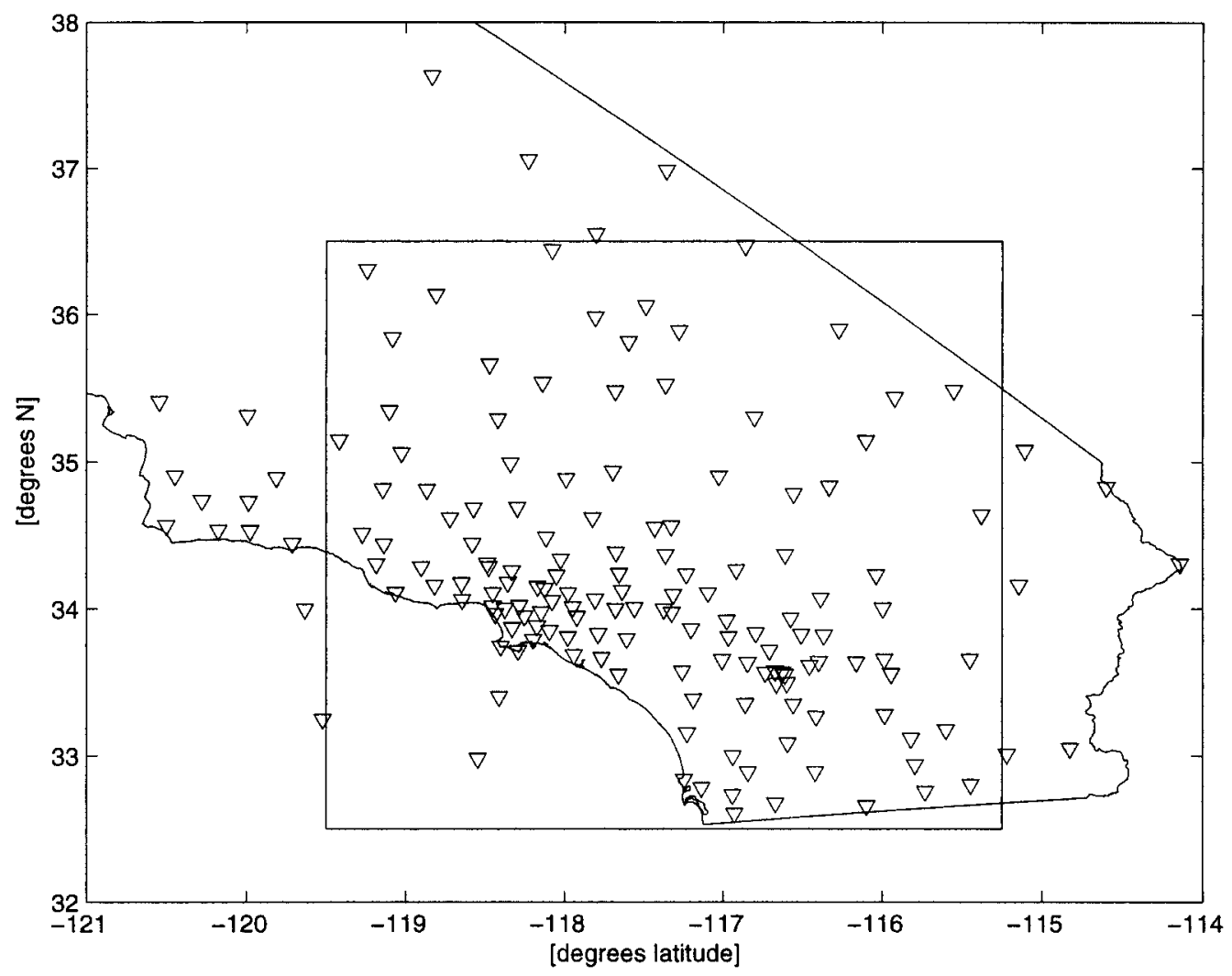

Figure 1. Station geometry for reconstruction tests. Trinet stations (upside-down triangles) are plotted over southern California. The square shows the boundary of the mesh area calculated. 


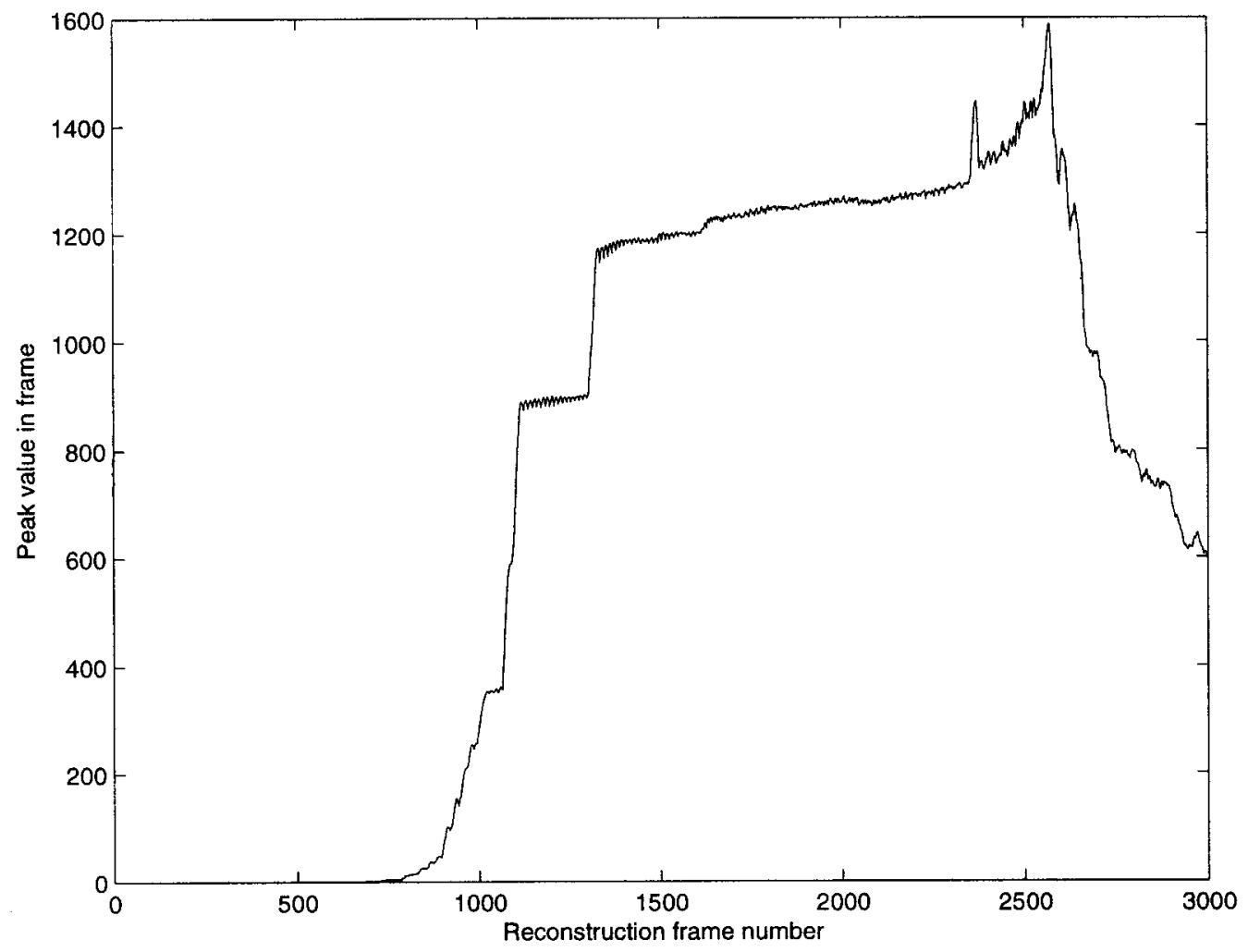

Figure 2. Maximum sum value for reconstruction of superimposed, time-shifted event. The maximum value at any point in the mesh is shown, over the 3000 time steps of the calculation. Two distinct peaks occur separated by the shift amount, 200 frames.

forms were calculated for each sensor location by summing the contributions from 51 Ricker wave simultaneous generating point events lying on a line from $(150,250) \mathrm{km}$ to $(250,250) \mathrm{km}$. A line of peak values falls on the expected location for the simulated events. The edges of the fault rupture are weakly located.

\section{Discussion}

Currently, broadband sensors in the Trinet array record ground motion every $0.05 \mathrm{sec}$. The code developed to test the RKL method is not optimized, and gains of sufficient magnitude to match the recording rate of the Trinet array should be achievable. The time elapsed between the event initiation and the reconstruction frame containing the corresponding peak varies with the maximum event-to-sensor distance. This time can be decreased by outputting the initial reconstruction with a set of sensors located within a smaller radius. For instance, the image reconstruction based only on the sensors most centrally located in the basin can be used to meet needs for near real-time location information. The contributions of sensors that are further away can easily be superimposed from a larger calculation.

Resolution of the solution can be improved by using a denser grid, higher-frequency signals, and more accurate velocity models. Simplified or incorrect velocity models change the travel times, possibly altering the reconstruction. Kao and Shan (2004) augment the SSA brightness sum with contributions from surrounding points in a chosen time window to account for variations in the velocity model. In the experiments performed here, a uniform velocity model of 6 $\mathrm{km} / \mathrm{sec}$ is applied. Known variations can be incorporated, such as the soft sediments in the basin region, with a $P$-wave velocity of less than $4.0 \mathrm{~km} / \mathrm{sec}$ (Hauksson, 2000). The modification suggested by Kao and colleagues is compatible with the RKL method presented here and could be tested to see whether it improves the location accuracy.

The reconstructions are calculated in three dimensions, with the mesh at depth divided into five $5-\mathrm{km}$ sections. In these tests the resolution is poor because of the small ratio of the depth of the actual events to the horizontal distance between the events and the sensors. This resolution can be improved by adding arrival times from structural reflections to the travel-time tables used in the reconstruction. Stronger peaks will occur at the appropriate depth when contributions from reflected arrivals are correlated. Husen et al. (2003) provide the rule of thumb that resolution of the event depth requires at least one station within a distance equal to 1.5 times the source focal depth.

The experiment using simulated data demonstrates the ability of the RKL method to locate a rupture trace. An actual rupture will take $40 \mathrm{sec}$ to propagate along the length of the 


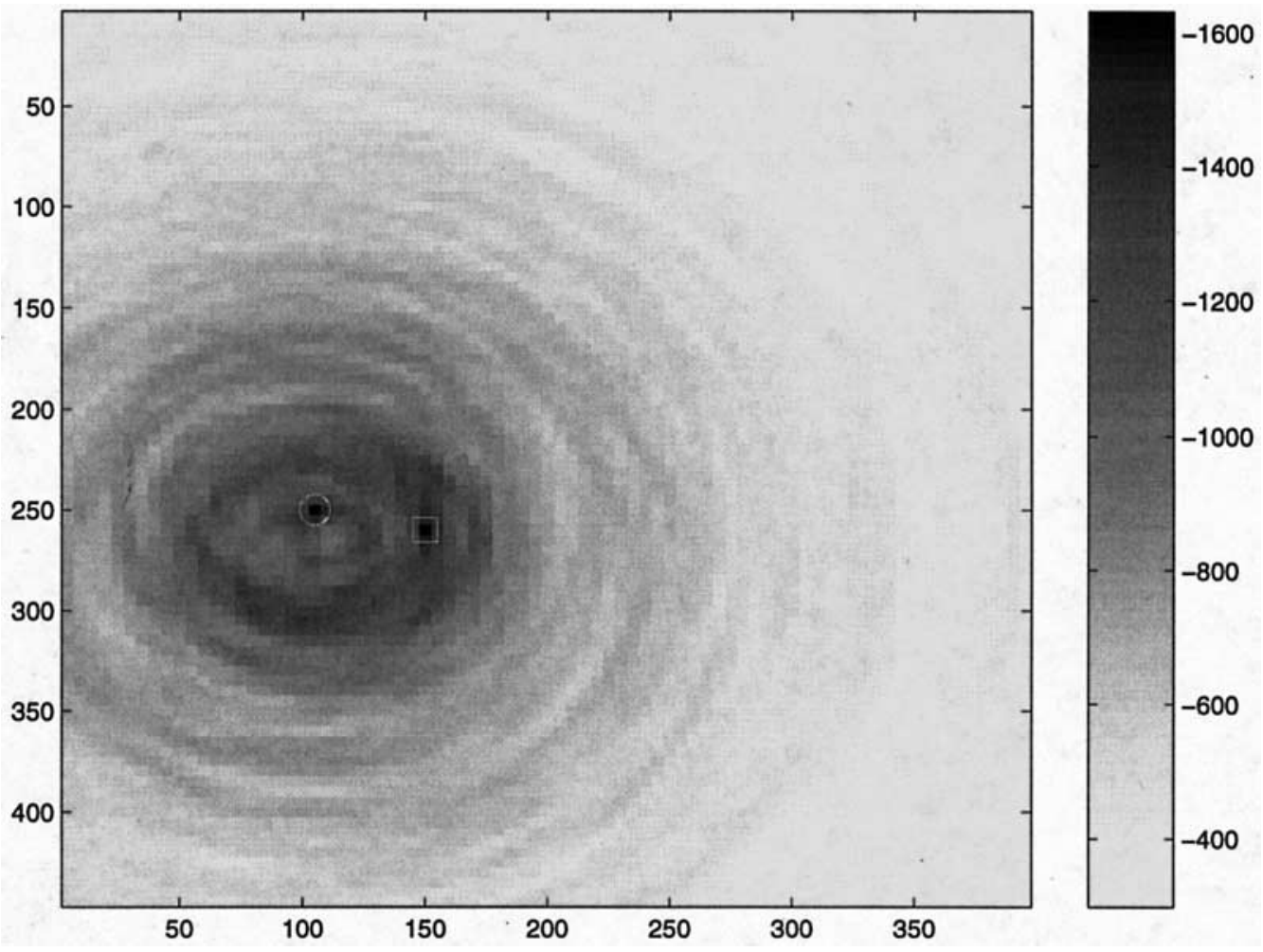

Figure 3. Peak image for space-shift test. Mesh coordinate $(0,0)$ corresponds to $(-119.5,36.5)$. The circle is centered on the real location of the original event. The square is centered on the expected location of the superimposed shifted event. The shading bar shows the value of the summation at each mesh location.

fault. This will result in a series of output frames showing a coherently propagating peak.

\section{Further Work}

Further work is required to implement the RKL method. Event identification is the most necessary development. Image-scanning methods will be implemented to robustly identify event locations. These algorithms will identify characteristics of the reconstruction when a true peak is found, such as a rapid increase in the value at a mesh point, as well as patterns corresponding to rupture traces and propagation with time. Under consideration for this purpose are methods such as deformable template matching (Burl, 2001; Burl et al., 2001), which can perform automatic detection of image features based on a few examples of the target signal. To enhance identification of the edges of a rupture, shape features can be incorporated in the scanning algorithms. By noting statistical differences between true and false peaks in example tests, the algorithms can be targeted more precisely. In the experiments described, true peaks have a significant persistence period, whereas false peaks jump around within the region of interest.

Additionally, artifacts of the method, like the rings seen in the time-shift experiment should be removable with a finite-difference technique. A real-time, image-based motion tracker can be overlain on the scanning algorithm. This can both rule out some false peaks, as they jump around, and identify the signatures of rupture motion along the fault. One candidate is the condensation algorithm (Isard and Blake, 1998).

Note that the magnitude of the peak in each solution is not relative to the magnitude of each event. Events that are poorly located or recorded within the sensor array will have smaller peaks due to contributions from fewer sensors, regardless of the size of the event. In further work, the input signals can be normalized to capture relative magnitude information.

The application of the Kirchhoff method to event-source location can be extended to apply full elastic theory. By summing the waveform values recorded in three directions and appropriately accounting for the projection angle from each sensor to each mesh location, a Kirchhoff reconstruction can calculate moment tensors over the entire mesh in real time. This is a direct extension of the current implementation. Increases in computational time can be mitigated by modifying the method to run on a parallel computing architecture. The image calculation is easily separated into different sections of the mesh or components of the recorded signal. 


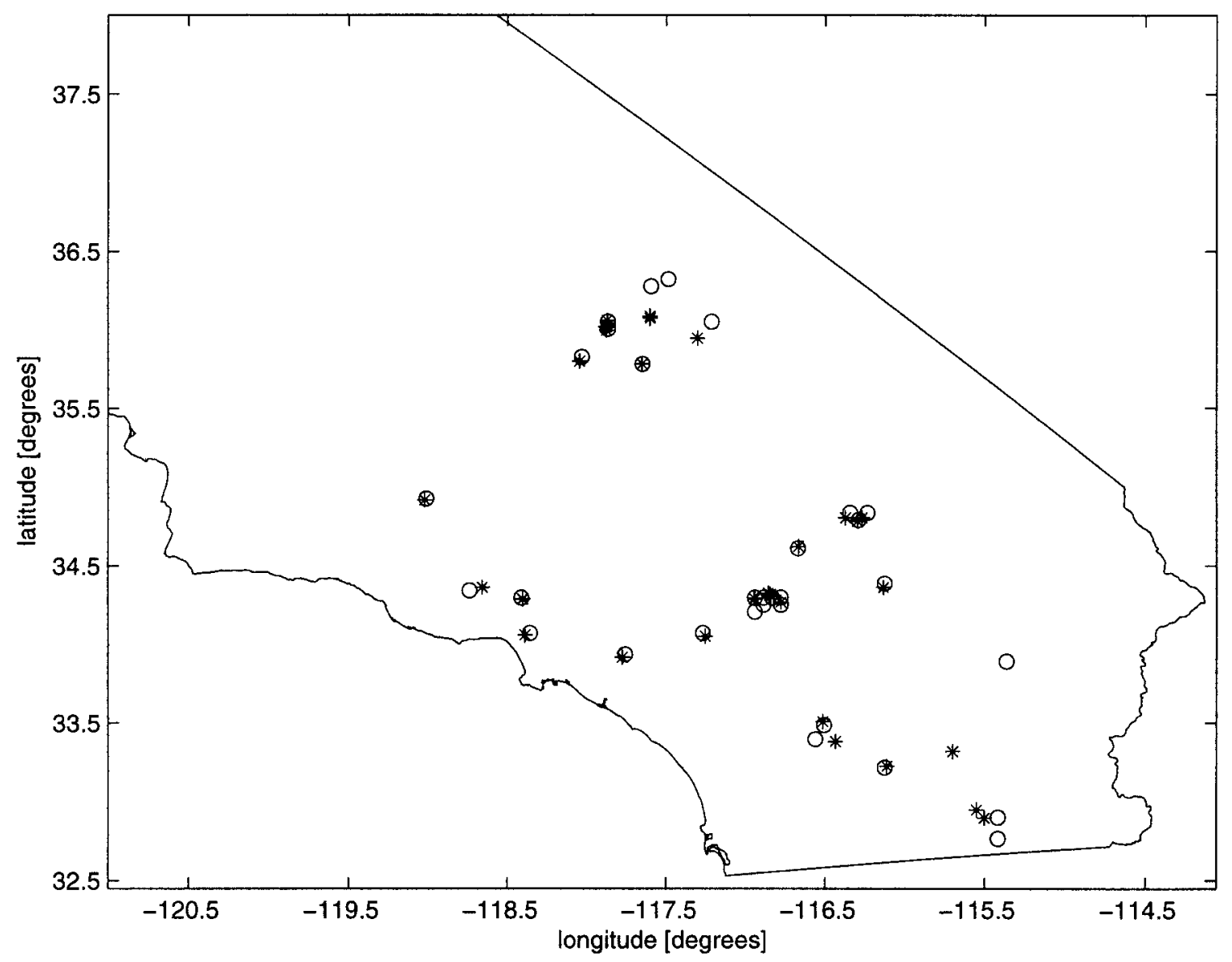

Figure 4. Comparison of expected and reconstructed locations. Real locations shown as black stars. Reconstructed locations are marked by circles.

Rapid source location within a seismic array can assist emergency services in allocating resources. Real-time information about the rupture trace is important to understand the stress effects on nearby faults, and related risk due to stress transfer. Future work by researchers may lead to the ability to forecast aftershocks, which will require accurate rupture traces (McCloskey, 2003). Rapid determination of the rupture surface can assist scientists studying the immediate, time-dependent deformation after an earthquake, allowing optimized targeting of campaign Global Positioning System (GPS) receivers and interferometric synthetic aperture radar (InSAR) satellites. In addition, the method can be used with early-warning, rapid-response systems. The RKL method will make it possible to use information from multiple stations. Several systems for early warning have been investigated and tested with seismic arrays in countries including Japan (Saita and Nakamura, 2003), Mexico (EspinozaAranda and Rodriguez, 2003), and Taiwan (Wu, 1999). The early-warning systems in Mexico and Taiwan estimate the event magnitude by using peak ground motion measurements caused by the $S$-wave arrivals. The Japanese system (UrEDAS) estimates both the magnitude and the event lo- cation based on $P$-wave arrivals, allowing slightly more warning time; however, this estimate is not based on all the sensors available. With seconds of early warning, precautions can be taken, such as automatic adjustments to building stiffness, shutdown of gas lines, and cessation of work with dangerous materials (Allen and Kanamori, 2003).

\section{Conclusion}

The Real-time Kirchhoff Location method is an alternative location method that is suited to real-time applications. It does not rely on picking and association processes. The method is robust because it provides a fixed computational load and it is not sensitive to interference by earthquakes occurring close together in space and time. With higher-resolution solutions, the method can identify rupture traces in addition to hypocenter locations. The method is designed to be incorporated into existing seismic arrays, and integration with the Southern California TriNet system is anticipated. In future work, the method will be extended to implement a fully elastic theory to calculate the instantaneous moment tensor at each point in a mesh. 


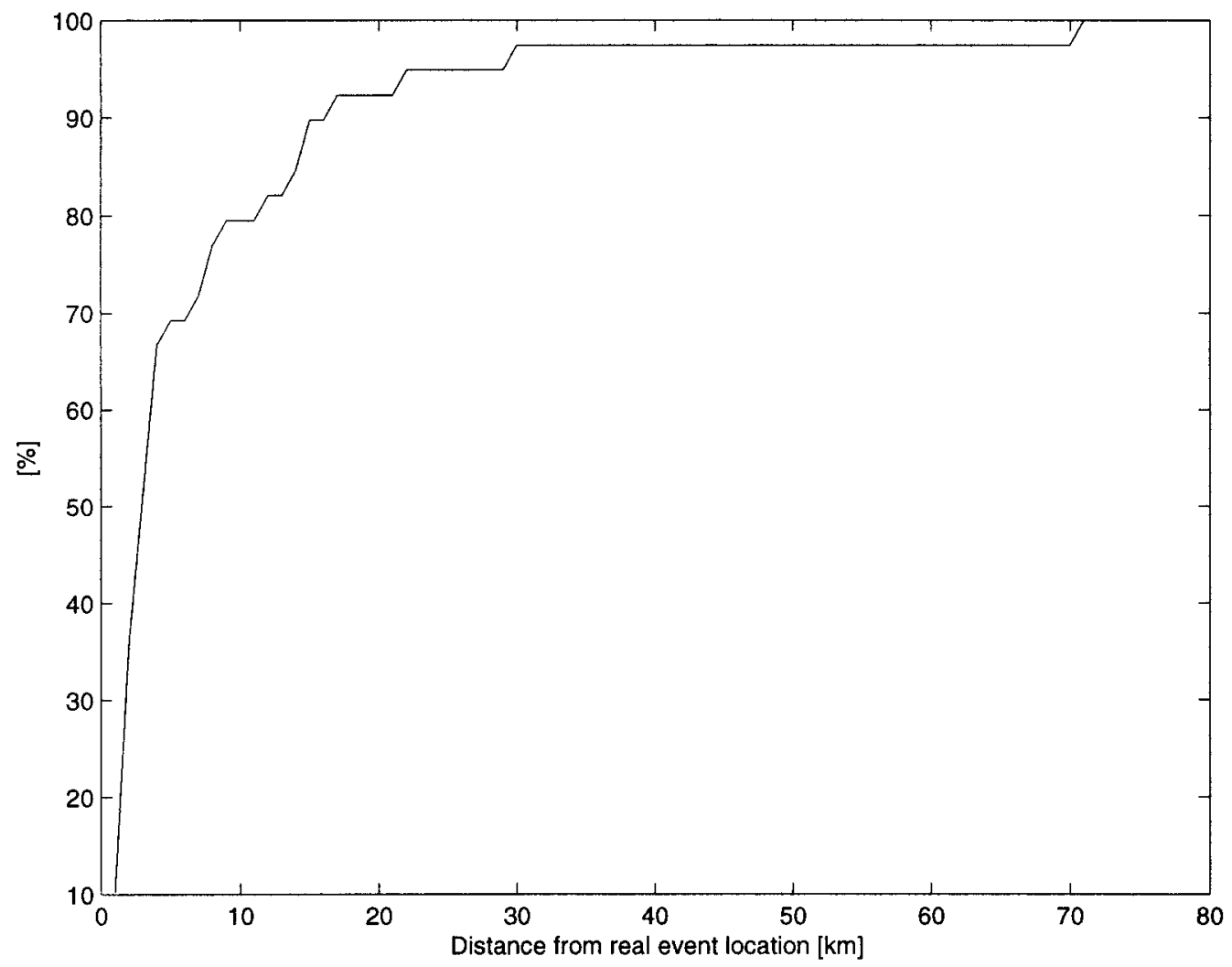

Figure 5. Percent of events reconstructed within a given distance of the real event location. The calculations are based on 40 tests using seismic data for southern California.

\section{Acknowledgments}

This research was carried out at the Jet Propulsion Laboratory, California Institute of Technology, under a contract with the National Aeronautics and Space Administration.

\section{References}

Allen, R. M., and H. Kanamori (2003). The potential for earthquake early warning in southern California, Science 300, 786-789.

Burl, M. C. (2001). Mining large image collections, in Data Mining for Scientific and Engineering Applications, R. Grossman, C. Kamath, V. Kumar, and R. Namburu (Editors), Massive Computing, Kluwer Academic Publishers, Norwell, Massachusetts.

Burl, M. C., W. J. Merline, W. Colwell, E. B. Bierhaus, and C. R. Chapman (2001). Automated detection of craters and other geological features, in Int. Symposium on Artificial Intelligence. Robotics and Automation for Space, Quebec, Canada, 18-22 June 2001.

Chang, W., and G. A. McMechan (1991). Wavefield extrapolation of body waves for 3-D imaging of earthquake sources, Geophys. J. Int. 106, 85-98.

Espinoza-Aranda, J., and F. Rodriguez (2003). The Mexican Sistema de Alerta Sismica (SAS) experience (abstract), Eos Trans. AGU 84, no. 46 (Fall Meet. Suppl.), S21B-02.

Hauksson, E. (2000). Crustal structure and seismicity distribution adjacent to the Pacific and North America plate boundary in southern California, J. Geophys. Res. 105, 13,875-13,903.

Husen, S., E. Kissling, N. Deichmann, S. Wiemer, D. Giardini, and M. Baer (2003). Probabilistic earthquake location in complex three-dimen- sional velocity models: application to Switzerland, J. Geophys. Res. 108, 1-12.

Isard, M., and A. Blake (1998). Condensation—conditional density propagation for visual tracking, Int. J. Comput. Vision 29, 5-28.

Jones, L. M., E. Hauksson, and A. F. Shakal (2003). TriNet: modern ground motion seismic network, in International Handbook of Earthquake and Engineering Seismology, W. H. K. Lee, H. Kanamori, P. C. Jennings, and C. Kisslinger (Editors), Academic Press, San Diego, 12751284.

Kao, H., and Shao-Ju Shan (2004). The source-scanning algorithm: mapping the distribution of seismic sources in time and space, Geophys. J. Int. 157, 589-594.

Lomax, A. (2000). Probabilistic earthquake location in 3D and layered models, in Advances in Seismic Event Location, C. Thurber and N. Rabinowitz (Editors), Kluwer Academic Publishers, Dordrecht, The Netherlands, 101-134.

McCloskey, J. (2003). Assessment of spatial aftershock probabilities: a feasibility study in earthquake hazard (www.errigal.ulst.ac.uk/ reports.html) (abstract). Eos Trans. AGU, 84, no. 46 (Fall Meet. Suppl.), S21B-02.

McMechan, G. A., J. H. Luetgert, and W. D. Mooney (1985). Imaging of earthquake sources in Long Valley caldera, California, 1983, Bull. Seism. Soc. Am. 75, 1005-1020.

Richards-Dinger, K. B., and P. M. Shearer (2000). Earthquake locations in southern California obtained using source-specific station terms, $J$. Geophys. Res. 105, 10,939-10,960.

Saita, J., and Y. Nakamura (2003) UrEDAS the early warning system for mitigation of disasters caused by earthquakes and tsunami, in Early Warning Systems for Natural Disaster Reduction, J. Zschau and A. N. Kuppers (Editors), Springer, Berlin, 453-460. 


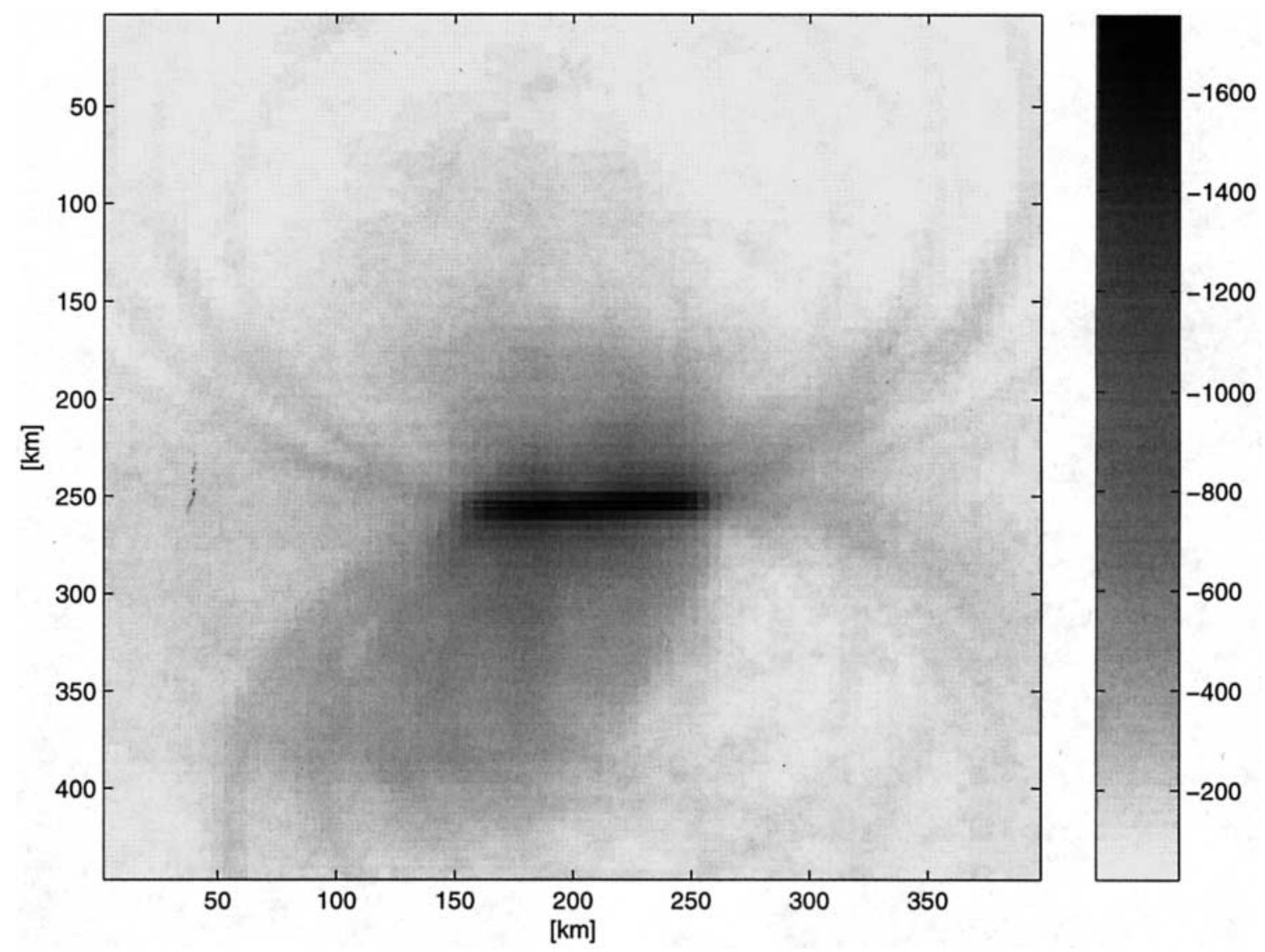

Figure 6. Peak value reconstruction image for simulated rupture event. The white line shows the extent of the original rupture. The rupture was simulated by Ricker wavelets emanating from an array of 51 points along the line.

Schneider, W. (1978). Integral formulation for migration in two and three dimensions, Geophysics 43, 49-76.

Southern California Earthquake Data Center (2004). www.data.scec.org. (last accessed March 2005).

Udias, A. (1999). Principles of Seismology, Cambridge University Press, Cambridge, United Kingdom.

Vidale, J. E., E. S. Cochran, H. Kanamori, and R. W. Clayton (2003). After the lightning and before the thunder; non-Omori behavior of early aftershocks? in American Geophysical Union Fall Meeting, San Francisco, California, 8-12 December 2003.

Waldhauser, F., and W. L. Ellsworth (2000). A double-difference earthquake location algorithm: Method and application to the northern Hayward fault, California, Bull. Seism. Soc. Am. 90, 1353-1368.

$\mathrm{Wu}$, Y. (1999). Development of an integrated earthquake early warning system in Taiwan: case for the Hualien area earthquakes, Terr. Atmos Ocean Sci. 10, 719-736.
Jet Propulsion Laboratory

California Institute of Technology

4800 Oak Grove Drive, M/S 300-233

Pasadena, California 91109-8099

(T.B.)

Jet Propulsion Laboratory

California Institute of Technology

4800 Oak Grove Drive, M/S 126-347

Pasadena, California 91109-8099

(R.G.)

California Institute of Technology

Seismological Laboratory

1200 East California Boulevard, M/S 252-21

Pasadena, California 91125-2100

(R.W.C.) 